\title{
HEMORRAGIA DE VÍAS DIGESTIVAS ALTAS DE URGENCIA EN EL HUN: CORRELACIÓN CLÍNICO- ENDOSCÓPICA
}

\author{
Upper digestive tract bleeding of emergency at huhmp: \\ clinic-endoscopic correlation
}

Fecha de recibido: 03-08-2010 • Fecha de aprobación: 15-10-2010

Resumen. La hemorragia aguda de vías digestivas altas (HVDA) es una de las emergencias más frecuentes en los servicios de urgencias ${ }^{(1)}$. Un reto importante para el clínico es evaluar que pacientes presentan un episodio hemorrágico que puede poner en riesgo la vida del paciente y sobre el que se debe actuar en forma interdisciplinaria con manejo médico, endoscópico y quirúrgico $^{(2,3)}$. El presente estudio muestra la pertinencia de la realización de endoscopias digestivas altas de urgencia, sus hallazgos endoscópicos y necesidad de manejo terapéutico. Se evaluaron los informes de 189 endoscopias realizadas con diagnóstico de HVDA de enero a junio de 2010 en la unidad de endoscopia digestiva del Hospital Universitario Hernando Moncaleano Perdomo (HUN). Se encontraron pacientes entre los 1 y 93 años, con predominio del género masculino (53\%). Encontramos al 60,4\% de los pacientes sin causa urgente de sangrado, el 39,6\% presentó causa urgente, de los cuales $29,1 \%$ con sangrado no variceal, y 10,5\% con sangrado variceal, se realizó terapéutica endoscópica a diez pacientes (5,1\%) con sangrado no variceal, con sangrado variceal se realizó terapéutica endoscópica a 17 (9\%). El diagnóstico más frecuente de las causas no urgentes de sangrado fue gastritis con un $26 \%$, seguido de ulcera gástrica con un $12 \%$. Los pacientes que ingresan al servicio de urgencias con diagnóstico de HVDA deben ser evaluados en forma ínte- gra con creación de protocolos para establecer cuales pacientes son de alto riesgo y ameriten la realización de una endoscopia digestiva alta de urgencia.

Palabras clave: tracto gastrointestinal superior, endoscopia gastrointestinal, úlcera estomacal, hemorragia gastrointestinal.

Abstract. Acute bleeding of upper digestive tract (ABUDT) is one of the most frequent emergencies at emergency services. Determining what patients have bleeding is an important challenge for clinicians since this it may be a risk for the patient's life. Bleeding requires an interdisciplinary approach including medical, endoscopic and surgical management.

This study shows the relevance of carrying out upper digestive endoscopies of emergency, its endoscopic findings and the need of therapeutic management. Reports of 189 endoscopies diagnosed as having ABUDT were evaluated, from January 1 to June 30, 2010 at the digestive endoscopy unit of Hernando Moncaleano Perdomo University Hospital.

There were patients aged from 1 to 93, predominantly male $(53 \%)$. Patients having no urgent bleeding cause was of $60,4 \%$, urgent cause was of $39,6 \%$ from which

\footnotetext{
* Cirujano Gastrooncología, Departamento de Cirugía, Facultad de Salud, Universidad Surcolombiana. Correo electrónico: rpino14783@gmail.com

** Estudiantes Programa de Medicina, Facultad de Salud, Universidad Surcolombiana.
} 
$29.1 \%$ had non-variceal bleeding and $10.5 \%$ had variceal bleeding. Endoscopic therapeutics was not performed on patients having non-variceal bleeding; on the contrary, endoscopic therapeutics was performed on $90 \%$ of patients having variceal bleeding. Gastritis $(26 \%)$ was the most frequent diagnosed cause of bleeding, following gastric ulcer $(12 \%)$. Patients admitted to emergency service having ABUDT should be evaluated in an integral form using protocols to determine high-risk patients that require to have an upper emergency digestive endoscopy.

Key words: upper gastrointestinal tract, endoscopy, gastrointestinal, stomach ulcer, gastrointestinal hemorrhage.

\section{INTRODUCCIÓN}

La Endoscopia de Vías Digestivas Altas (EVDA) es un examen invasivo que se realiza con criterios puntuales de urgencia, entre los que se encuentran Hemorragia de Vías Digestivas Altas; Ingestión de cáusticos y extracción de cuerpo extraño ${ }^{(4)}$. Estos criterios son actualmente utilizados como criterios de endoscopia de urgencia en el Hospital Universitario de Neiva, entendida como aquella que se realiza dentro de las primeras 24 horas de haber ingresado el paciente ${ }^{(5)}$. El servicio de endoscopia digestiva alta (EGD) como la mayoría de las unidades de los hospitales públicos cuenta con recursos limitado y volúmenes altos que conllevan a un represamiento en la oportunidad de atención, es por esto que se hace imperioso realizar estudios que lleven a tratar de evaluar que pacientes realmente se benefician de este procedimiento realizado en forma urgente ${ }^{(6)}$.

La edad, estado circulatorio al ingreso, comorbilidad, signos clínicos de hemorragia reciente representa criterios que pueden ser utilizados como factor pronóstico, en cuanto a que pacientes requieren o no EGD de urgencia. (Escala de Rockall) $)^{(7)}$.

El presente estudio tuvo como finalidad evaluar la pertinencia en la solicitud de EVDA de urgencia en la unidad de endoscopia del Hospital Universitario Hernando Moncaleano Perdomo de Neiva durante el periodo de 1 de enero a 31 de junio de 2010. Describir las características socio-demográficas de los pacientes que ingresan con diagnostico presuntivo de HVDA. Valorar la necesidad clínica y endoscópica de EVDA de urgencias. Conocer los diagnósticos endoscópicos más frecuentes reportados por EGD. Describir los métodos terapéuticos más usados para el tratamiento de HVDA.

\section{MATERIALES Y MÉTODOS}

El presente proyecto de investigación es un estudio descriptivo, de prevalencia, retrospectivo que se llevó a cabo por medio de resultados obtenidos en los informes de EGD de urgencia en los pacientes a quienes se les realizó diagnóstico presuntivo de HVDA en el servicio de endoscopia digestiva del HUN durante el periodo de 1 de enero hasta el 30 de junio de 2010 (figura 1).

Se revisaron 1253 resultados de EVD. 973 correspondían a EVDA. De estas se descartaron 784 y se seleccionaron 189, que cumplían con los criterios de inclusión, los cuales fueron:

- Pacientes con diagnóstico presuntivo de hemorragia de vías digestivas altas.

- Atendidos en el HUN.

- Procedimiento de EGD realizado en la unidad de endoscopia del Hospital Universitario de Neiva, durante el periodo de 1 de enero a 30 de junio de 2010 .

\section{RESULTADOS}

Los pacientes más afectados están en un rango de edad de 65 a 85 años (figura 2). El servicio de urgencia más solicitante de EGD, fue el de observación de adultos (figura 3). De los 189 pacientes, en $114(60,4 \%)$ no se encontraron lesiones endoscópicas causantes de sangrado digestivo. En los restantes $75(39,6 \%)$ pacientes, 20 presentaron sangrado de origen variceal y $55(29,1 \%)$ pacientes presentaron sangrado de origen no varicial.

En el grupo de lesiones no causantes de sangrado los diagnósticos principales fueron: Gastritis, úlcera gástrica no sangrante, esofagitis, duodenitis, endoscopias normales (Figuras 4-6).

En el grupo de lesiones causantes de sangrado de origen no varicial, se encontraron $5(2,6 \%)$ pacientes con úlcera Forrest tipo I, 10 (5,3\%) Forrest tipo II y $22(11,6 \%)$ con úlceras Forrest tipo III (figura 7).

En cuanto al tipo de terapéutica endoscópica, 44 (23,2\% del total) de 55 pacientes con sangrado no variceal recibieron algún tipo de tratamiento. $6(3,17 \%)$ pacientes recibieron escleroterapia, 2 (1\%) tuvieron manejo con clips, 2 (1\%) recibieron 


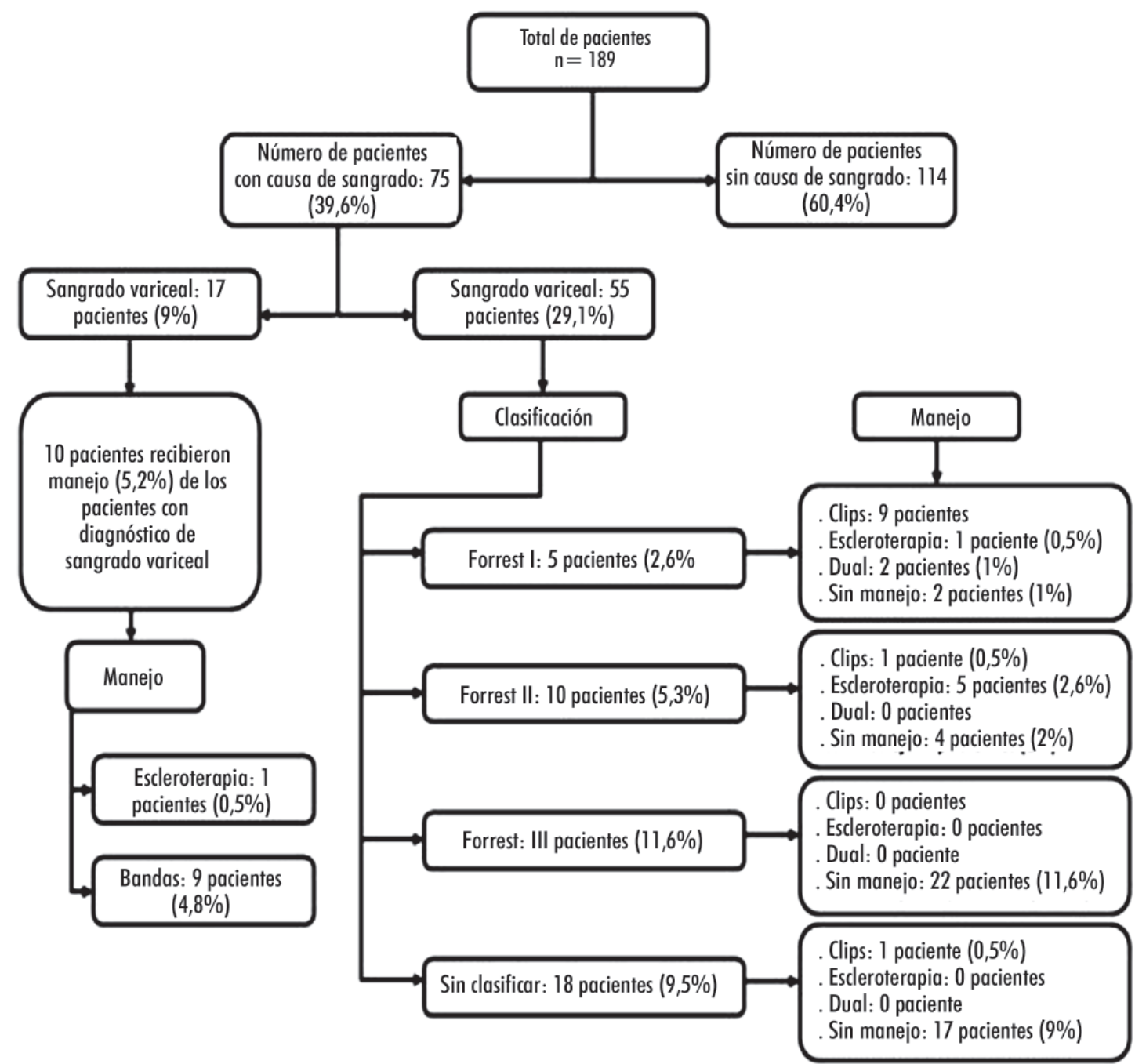

Figura 1. Algoritmo del estudio de hemorragia de vías digestivas altas de urgencia en el HUHMP: correlación clínico-endoscópico, de 1 de enero a 30 de junio de 2010, en el Hospital Universitario de Neiva.

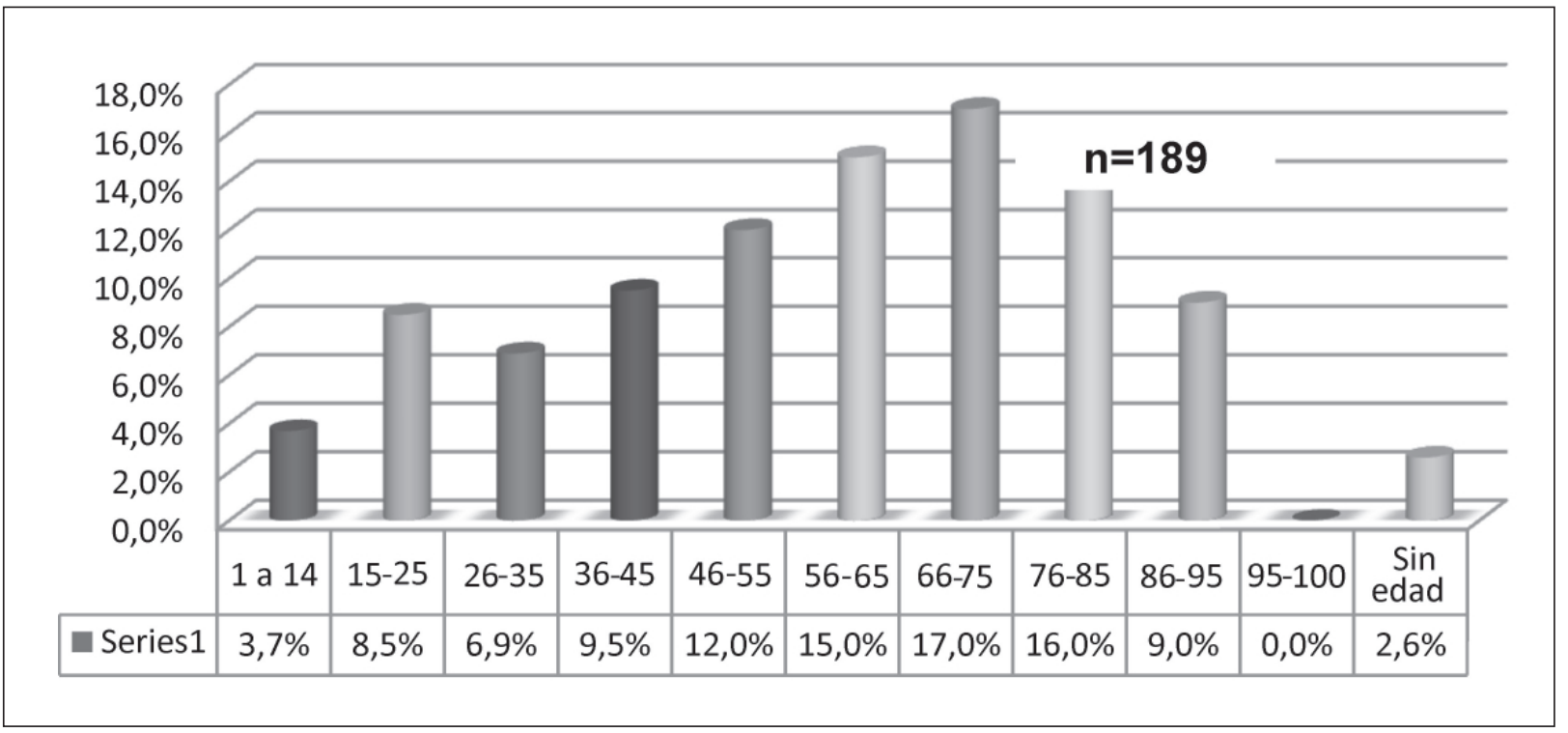

Figura 2. Distribución de la edad en el momento del diagnóstico de los pacientes con hemorragia de vías digestivas altas, en el Hospital Universitario de Neiva enero- junio 2010. 


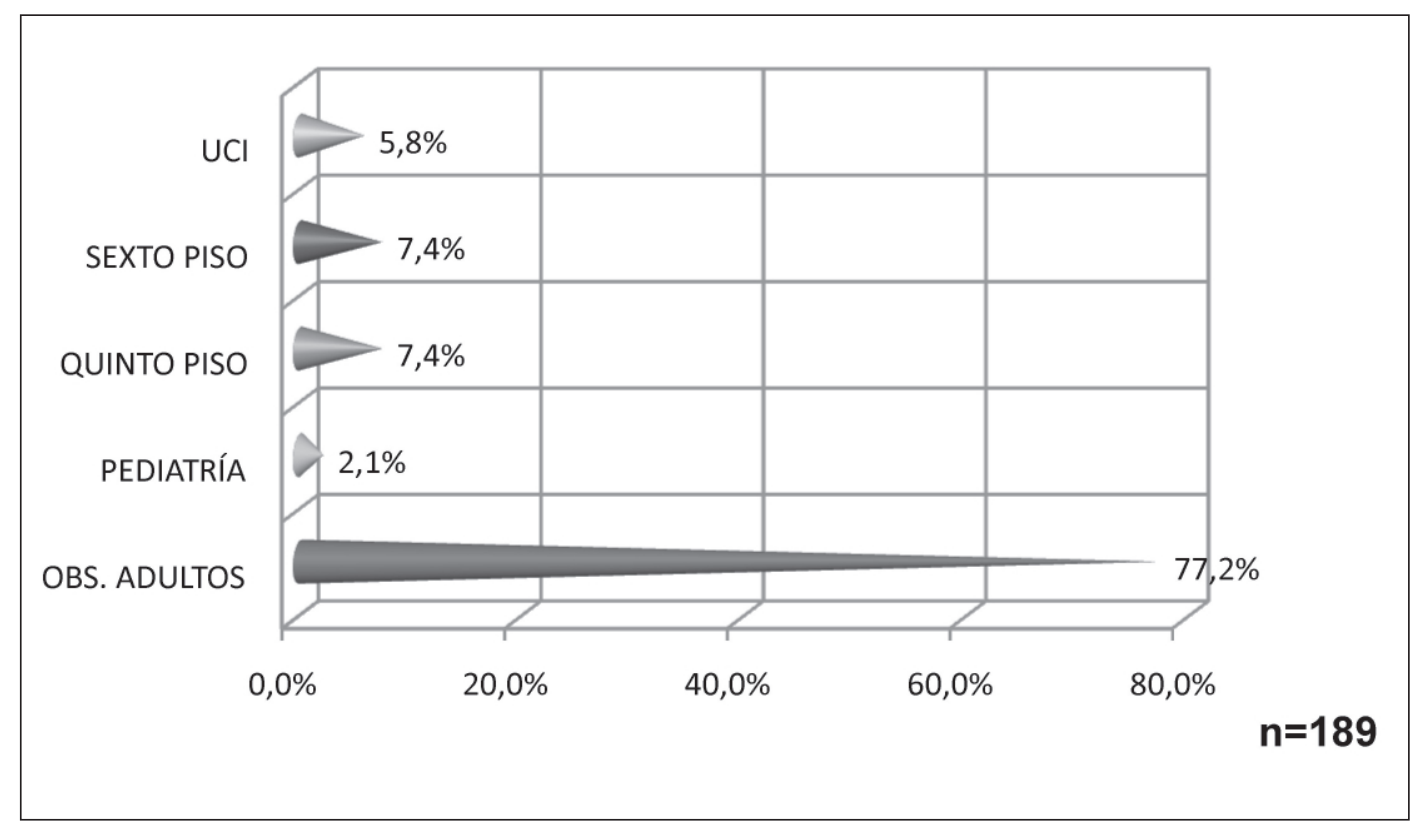

Figura 3. Servicio solicitante de EGD de urgencia con diagnóstico de hemorragia de vías digestivas altas, en el Hospital Universitario de Neiva enero-junio 2010.

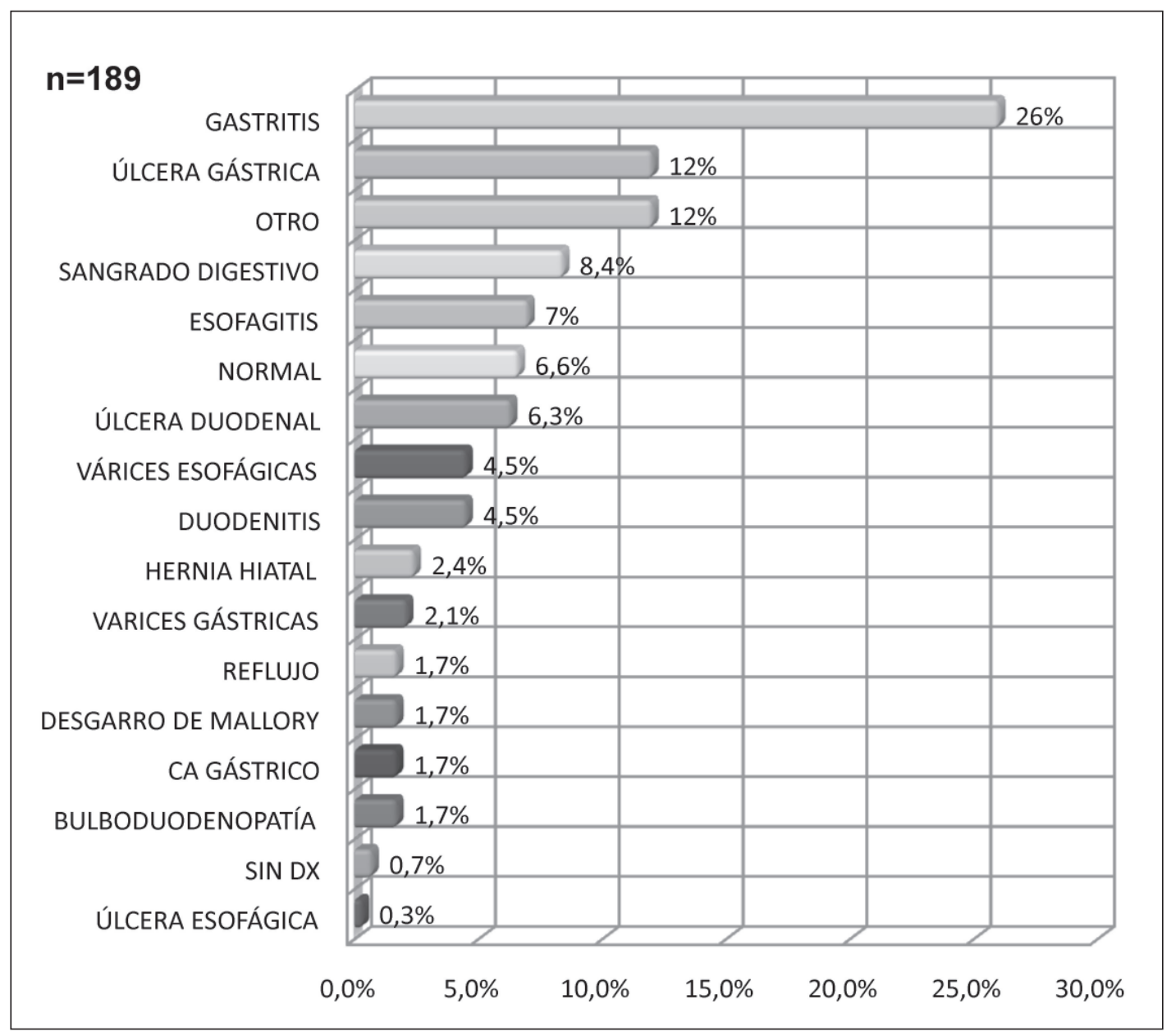

Figura 4. Diagnósticos encontrados en la EGD de urgencia con diagnóstico de hemorragia de vías digestivas altas, en el Hospital Universitario de Neiva enero-junio 2010. 


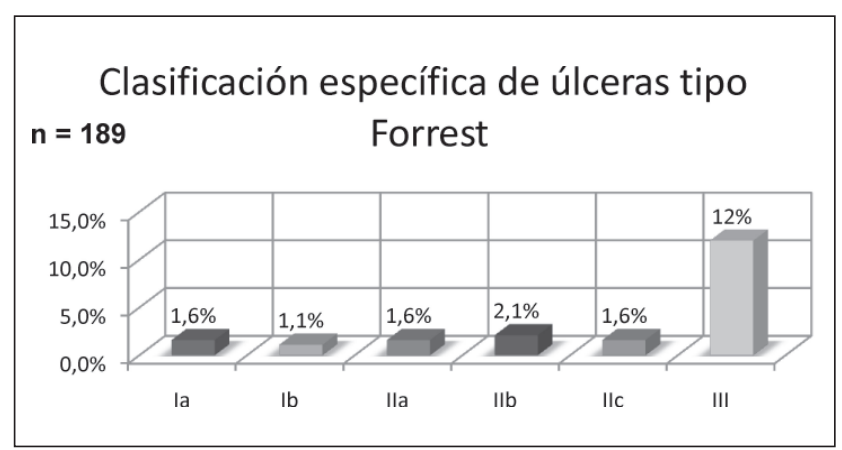

Figura 5. Clasificación específica de úlceras tipo Forrest en la EGD de urgencia con diagnóstico de hemorragia de vías digestivas altas, en el Hospital Universitario de Neiva enero-junio 2010.

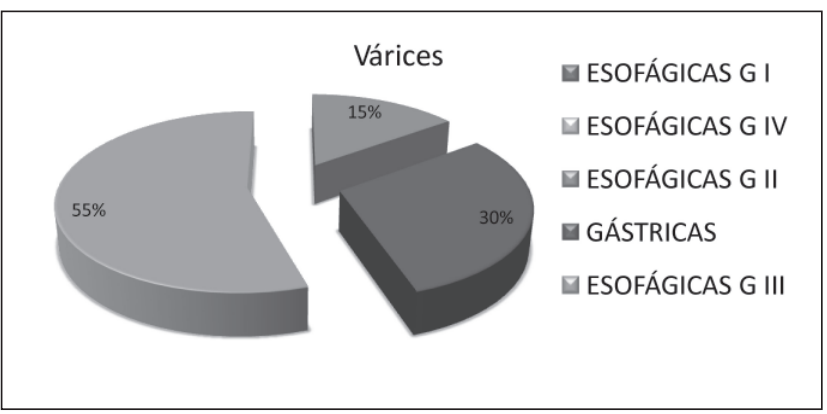

Figura 6. Tipo de várices halladas en la EGD de urgencia con diagnóstico de hemorragia de vías digestivas altas en el Hospital Universitario de Neiva enero-junio 2010.

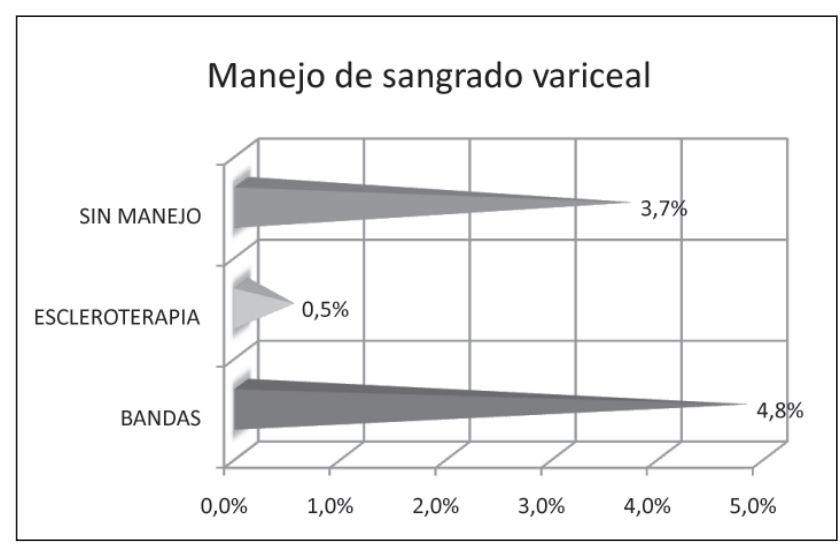

Figura 7. Manejo del sangrado variceal durante la realización de EGD de urgencia en el Hospital Universitario de Neiva enero-junio 2010.

terapia dual que consiste en escleroterapia más clips y $34(17,9 \%)$ pacientes no tuvieron ningún tipo de manejo.

En los pacientes con sangrado de origen varicial (17 pacientes) la gran mayoría presentaron várices grado III, (Figura 7), recibiendo manejo 10 de ellos, 9 con bandas de caucho y uno con escleroterapia, en los 7 restantes no se realizó terapéutica (figura 7).

Dentro de los pacientes a los que se les realizó endoscopia de urgencia y no se les realizó clasificación del sangrado en el momento del procedimiento por algún motivo, solo $1(0,5 \%)$ paciente recibió terapia endoscópica con clips.

\section{DISCUSIÓN}

La hemorragia digestiva alta continúa siendo una patología de alta frecuencia en los servicios de urgencias de los hospitales ${ }^{(1)}$. Los servicios de endoscopia presentan generalmente alta demanda de servicios, la endoscopia digestiva alta es un examen invasivo, no libre de complicaciones $^{(8)}$.

El presente estudio encontró un grupo importante de solicitudes de EGD con diagnóstico clínico de hemorragia digestiva alta, cuyo diagnóstico final no fue significativo $(60,4 \%)$. El $39,6 \%$ de los pacientes presentaron causas significativas de sangrado, en otras palabras encontramos correlación clínico-endoscópica. La tasa de terapéutica endoscópica fue baja, pensamos que es debido a la falta de elementos terapéuticos en la realización del mismo.

Los reportes de la literatura informan que estos pacientes deben ser sometidos a protocolos de manejo en hemorragia digestiva, los cuales han demostrado mejoría en la morbimortalidad general y disminución en los costos y estancia hospitalaria ${ }^{(9)}$.

Sugerimos que en los hospitales existan protocolos de manejo en esta patología para determinar la necesidad de solicitar EGD de urgencia que ayudaría a disminuir costos al sistema de salud y además evitar congestiones innecesarias en las listas de espera de los servicios de gastroenterología.

\section{CONCLUSIONES}

A pesar de indicaciones claras de realización EGD de urgencias, en este estudio se encontró una gran población de pacientes con solicitudes no indicativas de este procedimiento de urgencia, lo cual sugiere que la mayoría de pacientes no tenían una indicación endoscópica para la solicitud de EGD. Con esto se pretende incitar a cambios en los diferentes servicios de 
los hospitales, que en el futuro eviten remisiones innecesarias a los servicios de gastroenterología, aumentos de gastos del sector salud y congestión de este servicio, por lo cual, se recomienda realizar un segundo estudio donde se evalué por más tiempo la necesidad clínico- endoscópica de una solicitud de EGD.

\section{REFERENCIAS}

1. Kaviani , M.J., Pirastehfar, M., Azari, A. et al. 2010. Etiology and outcome of patients with upper gastrointestinal bleeding: A study from South of Iran. Saudi Journal of Gastroenterology 16:253-259.

2. Kasem, A.M., Kamal, T., Chandra, N.N. et al. 2006. Management of acute upper gastrointestinal bleeding in a district hospital. Journal of Laparoendoscopic \& Advanced Surgical Techniques A. 16(4):355-361.

3. Olokoba, A.B., Olokoba, L.B., Jimoh, A.A. 2009. Upper gastrointestinal tract bleeding in Ilorin, Nigeria-a report of 30 cases. Nigerian Journal of Clinical Practice 12(3):240-244.
4. Trujillo, O.E., Navarro, A.M., Guerrero, M.M. 2007. Uso apropiado de la esofagogastroduodenoscopia diagnóstica y su relación con hallazgos positivos. Revista médica del Instituto Mexicano del Seguro Social 45(1):83-87.

5. Joshua, G., Alan, B. 2010. A summary of recent recommendations on the management of patients with nonvariceal upper gastrointestinal bleeding. Pol Arch Med Wewn 120(9):341-346.

6. Kwun, C., Dong, H.L., Heung, U.K. et al. 2009. Guidelines of Treatment for Bleeding Peptic Ulcer Disease. Korean Journal of Gastroenterology 54(5):298-308.

7. Vreeburg, E.M., Terwee, C.B., Snel, P. et al. 1999. Validation of the Rockall risk scoring system in upper gastrointestinal bleeding. Gut 44(3):331-335.

8. Ernst, J.K. 2010. Endoscopy: Risk assessment in upper gastrointestinal bleeding. Nature Reviews Gastroenterology and Hepatology 119:480-482.

9. Sarin, N., Monga, N., Adams, P. 2009. Time to endoscopy and outcomes in upper gastrointestinal bleeding. Canadian Journal of Gastroenterology 23(7):489-493. 